\title{
A Multidisciplinary Workplace Intervention for Chronic Low Back Pain among Nursing Assistants in Iran
}

\author{
Sarallah Shojaei ${ }^{1,2}$, Sedigheh Sadat Tavafian ${ }^{1}$, Ahmad Reza Jamshidi ${ }^{3}$, Joan Wagner ${ }^{4}$ \\ ${ }^{1}$ Department of Health Education, Faculty of Medical Sciences, Tarbiat Modares University, Tehran, Iran \\ ${ }^{2}$ Nekoei Hospital, Qom University of Medical Sciences, Qom, Iran \\ ${ }^{3}$ Rheumatology Research Center, Tehran University of Medical Sciences, Shariati Hospital, Tehran, Iran \\ ${ }^{4}$ Faculty of Nursing University of Regina, Regina, SK, Canada
}

Study Design: Interventional research with a 6-month follow-up period.

Purpose: We aimed to establish the effectiveness of a multidisciplinary workplace intervention on reduction of work-related low back pain (WRLBP), using ergonomic posture training coupled with an educational program based on social cognitive theory.

Overview of Literature: WRLBP is a major occupational problem among healthcare workers, who are often required to lift heavy loads. Patient handling is a particular requirement of nurse aides, and has been reported as the main cause of chronic WRLBP.

Methods: We included 125 nursing assistants from two hospitals affiliated to Oom University of Medical Sciences from May to December 2015. There was an intervention hospital with a number of 63 nursing assistants who received four multidisciplinary educational sessions for 2 hours each plus ergonomic posture training over two days and a control hospital with a number of 62 nursing assistants who didn't receive educational intervention about low back pain. The outcomes of interest were reductions in WRLBP intensity and disability from baseline to the follow up at 6 months, which were measured using a visual analog scale and the Quebec Disability Scale. Descriptive and analytical statistics were used to analyze the data.

Results: The comparison tests showed significant change from baseline in reduction of WRLBP intensity following the multidisciplinary program, with scores of $5.01 \pm 1.97$ to $3.42 \pm 2.53$ after 6 months on the visual analog scale in the intervention group $(p<0.001)$ and no significant change in control groups. There was no significant difference in the disability scores between the two groups $(p=0.07)$.

Conclusions: We showed that our multidisciplinary intervention could reduce the intensity of WRLBP among nurse aides, making them suitable for implementation in programs to improve WRLBP among nursing assistants working in hospitals.

Keywords: Work-related low back pain; Multidisciplinary program; Ergonomic approaches; Nurses' aides; Randomized controlled trial

\section{Introduction}

Low back pain is a major occupational problem among healthcare workers [1], with work-related low back pain (WRLBP) being a costly occupational health problem that is a major cause of disability-related absenteeism

\footnotetext{
Received Aug 17, 2016; Revised Dec 4, 2016; Accepted Dec 13, 2016

Corresponding author: Sedigheh Sadat Tavaian

Department of Health Education, Faculty of Medical Sciences, Tarbiat Modares University,

No 212, Tehran P.O. Box 14115-331, Iran

Tel: +98-21-82884547, Fax: +98-21-82884555, E-mail: tavafian@modares.ac.ir
} 
influenced directly by work-related factors [2]. The lifetime prevalence of reported low back pain among hospital workers varies between countries, ranging from $39 \%$ in Hong Kong [3] to $76 \%$ in the Netherlands [4]. Lifting of heavy loads is a key duty of many healthcare practitioners [5], but among nurse aides, patient handling is the most prevalent duty and has been reported as the main cause of WRLBP [6]. It has been argued that performing more than 10 patient-handling activities per day increases the risk of WRLBP aggravation among female nursing assistants [7].

One way to prevent chronic back pain is to understand the principles of proper body mechanics and to learn how to maintain correct ergonomics and vertebral posture during daily activities [8]. In developed countries, significant improvements in staff behaviors and in creating a supportive environment have helped prevent back pain [9]. Although studies have shown that ergonomics training to maintain an adequate body posture at work can reduce the prevalence of work-related musculoskeletal disorders among nurses [10], few studies have focused on changing risk behaviors (e.g., not maintaining correct vertebral posture), few have based their research on interventional models or theories, and few have focused on the course of low back pain after an intervention [10]. Indeed, as stated by Miller and Albert Bandura-the influential psychologists responsible for social cognitive theory-one only learns behaviors through observation, modeling, and motivation (e.g., positive reinforcement and rewards).

Many Iranian nursing assistants are not properly educated about the potential occupational hazard of WRLBP or about how to control and prevent such musculoskeletal disorders. Moreover, nursing assistants are at particularly high risk of back pain, and few studies have focused on the issue of WRLBP in this group. In this study, we therefore aim to investigate the effects of an educational program (based on social cognitive theory) on pain severity and disability reduction among nursing assistants with WRLBP.

\section{Materials and Methods}

\section{Study design}

This was an interventional study with follow-up period of 6 months. We used random sampling to select 125 nursing assistants working in two hospitals affiliated to Qom University of Medical Science, Iran, between May 2015 and December 2015. The study was approved by the ethi- cal committee of Tarbiat Modares University.

\section{Study population}

The inclusion criteria were as follows: working as a nurse aide for at least one year, aged 20 to 60 years, suffering from lower back pain for at least 3 months, and having that back pain diagnosed based on a physician's examination. The exclusion criteria were as follows: a history of vertebral surgery, participation in other interventional approaches for back pain during the study period, any neck or lumbar disease, any congenital abnormalities or tumors of the spine, pregnancy, and lack of consent. No paraclinical tests were used to diagnose chronic low back pain or assess the exclusion criteria.

We randomly selected two hospitals affiliated to Qom University of Medical Sciences from all hospitals in Qom, and then randomly allocated them to be either the control or the intervention hospital. The work conditions and characteristics of both hospitals were the same, and had a similar status and procedure for patient admission and hospitalization. We enrolled 63 and 62 eligible nursing assistants from the intervention and control hospitals, respectively. The groups were matched by age, gender, educational level, and occupation.

Participants in the intervention group received the specified educational program, and those in the control group were left to continue their practice as usual. The procedure and aims of the study were explained to all potential participants, and they were only asked to sign the consent form if they were satisfied. There was no loss to follow up, because all nursing assistants continued to work in the hospital and the first researcher maintained contact throughout the study. Full contact details, including telephone number (home and work), mobile number, e-mail address, were obtained at inclusion in the study.

\section{Intervention}

The multidisciplinary intervention consisted of an educational program and ergonomic posture training. The educational program was based on four concepts of social cognitive theory that have been shown to predict healthy behaviors among nursing assistants suffering from low back pain. The procedure used to determine these predictors has been described elsewhere [11]. Because of the nature of the intervention, we could not mask the partici- 
pants or treatment providers to the treatment assignment, but blinding was used for the data analyses. The educational program included the following four sessions, each lasting 2 hours.

\section{1) Promoting self-efficacy for compliance with an ergo- nomic posture}

The concept of self-efficacy points to the belief of a person's ability to perform behaviors that bring desired outcomes [12]. Therefore, a health education specialist (first author) educated participants to be confident about the correct ergonomic posture by addressing and practicing the skills needed to maintain a correct biomechanical posture of vertebra. In this session, step-by-step education about how to maintain the correct/natural postures of the vertebrae while standing, sitting, walking, and reclining in the workplace was provided. Back-strengthening exercises were also taught. At the same time, the educator aimed to improve the nurse aides' beliefs about their ability to comply with this new information when transferring or repositioning patients in clinical situations. Mastery of the intended skills was assessed and ensured, and people in similar conditions who engaged in such behaviors in their workplace were set as role models. Proper practices were demonstrated by an instructor, who then asked the participants to copy; there was a focus on asking individuals to concentrate on preventive behaviors for backache. The need to be able to ask others for assistance was stressed when patient lifting was needed, and all participants were reassured that this was even appropriate under critical conditions.

The following educational methods were applied: guided practice, confirmation of skillful experience, verbal persuasion, encouragement, improvement in mental-physical states, training ascription, behavior selfmonitoring, creation of conditional rewards, goal setting, benchmarking, skill training, task setting by difficulty and response, negotiation, stress reduction techniques, and role-playing. These techniques and methods were chosen to increase self-efficacy in patient carriers. To increase self-efficacy, both factual and conceptual barriers blocking an individual's ability to complete a task were addressed. Short- and long-term targeting was carried out, and ascription training was given. Those who regarded the reason behind their failure to be their inability or poor fortune were less likely to be successful, so previous unfruitful attempts were ascribed to external factors.
2) Promoting self-regulation for compliance with an ergonomic posture

Self-regulation involves controlling oneself through selfmonitoring, goal setting, feedback, self-reward, selfinstruction, and enlistment of social support [12]. In this session, we educated the participants on how they could comply with the correct ergonomic posture at work and use self-control skills to resolve problems. The fist author provided education about self-monitoring/self-management for maintaining ergonomic vertebrae and correct back posture when transferring or repositioning patients, as well as when standing, sitting, walking, and reclining. There was a focus on reviewing the correct and incorrect behaviors for preventing back pain at the end of each shift or before bedtime. Self-assessment during patient handling was practiced with the participants, and each participant was asked to develop an action plan to help them prevent low back pain behaviors in their workplace.

Individuals were asked to monitor themselves during a work shift, assigning positive or negative scores to themselves after reviewing proper or improper practices at the end of each shift or before bed. They were asked to reduce their negative scores and increase their positive ones. In addition, individuals were asked to arrange appropriate preventive backache and spinal cord plans in workplaces and to achieve inclusive dominance over their responsibilities. By comparing their own behaviors to cited behaviors, individuals could express appropriate emotional reactions

3) Highlighting outcome expectations when maintaining an ergonomic posture

The concept of outcome expectations points to beliefs about the likelihood and value of the consequences of behavior choices [12]. In this session, we emphasized the positive outcomes of complying with correct ergonomic posture and of maintaining an ergonomic posture of the vertebrae when transferring/repositioning the patients, standing, sitting, walking, and reclining. These outcomes included reducing pain severity, improving physical/mental function, reducing healthcare costs, reducing work absenteeism, and increasing satisfaction with work.

4) Focusing on emotional coping when maintaining an ergonomic posture in the workplace

Emotional coping refers to the techniques employed by a person to control emotional and physiological states as- 
sociated with a new condition/behavior [12]. Emotionfocused coping involves trying to reduce the negative emotional responses associated with stress, such as those that arise from needing to maintain an ergonomic vertebral posture in the workplace. Therefore, we taught participants how to cope emotionally with the necessary changes, emphasizing the potential to feel satisfaction, happiness, and excitement from maintaining their vertebrae during patient handling or other duties. The potential benefits of senses of worth, philanthropy, and humanitarianism while handling/transferring patients were focused on to emphasize preventing damage to oneself and others. All educational classes were managed through group discussions and question-and-answer procedures.

\section{Measurements}

Outcomes were assessed using questionnaires. First, demographic data were collected at baseline, including age, body mass index, gender, education level, and WRLBP and its characteristics (e.g., sciatica pain). Second, a studyspecific questionnaire was completed covering prevention behaviors related to back pain, which also included a visual analog scale and a Quebec Disability Scale for assessing WRLBP intensity and disability, respectively. Questionnaires were completed by participants of both the intervention and control groups at baseline (before intervention) and at follow up after 6 months. The details of the latter three questionnaires are as follows.

\section{1) The behavior questionnaire}

This included 12 questions regarding preventive behaviors for low back pain that were adopted from the Occupational Safety and Health Administration guidelines for nurses [13]. The questions focused on the following: the correct steps for moving and handling patients, using principles of body mechanics when moving or lifting patients, correct bending over the patient or a work surface, applying the correct posture while standing or sitting, and using appropriate work management activities to reduce exposure to WRLBP risk factors. We employed a 4-point Likert scale (never, 1; sometimes, 2; often, 3; and always, 4) per question, giving a total score range of $12-48$, with lower scores indicating the worst vertebral positions during activity. Respondents were free to take their time to answer questions, but did so under the researcher's supervision. Based on the consensus of an expert panel, scores for this questionnaire were categorized as poor (12-24), average (25-36), or acceptable (37-48). To assess the questionnaire's validity, an expert panel of 10 specialists checked the items and provided recommendations (two neurosurgeons, one rheumatologist, one epidemiologist, three nursing teachers, and three health education experts). After assessing the content validity, the questionnaire was simplified so that each participant could answer the questions easily. These procedures have been described in a previous study [11]. A pilot study was conducted among 25 nursing assistants from different units in Qom hospitals to determine the face validity of each question. Based on this, items were evaluated and modified for appearance, fitness, brevity, clarity, ease of understanding, and inclusiveness.

\section{2) The visual analog scale}

We used a visual analog scale to measure the intensity of low back pain. This is a one-dimensional self-completed measure in which the respondent is asked to place a point on a $10 \mathrm{~mm}$ line that best represents his or her pain intensity [14]. A higher score indicates greater pain intensity. Based on the distribution of scores on the pain visual analog scale among postsurgical patients who described their pain intensity as none, mild, moderate, or severe, the following cutoff points were used: no pain $(0-4 \mathrm{~mm})$, mild pain $(5-44 \mathrm{~mm})$, moderate pain $(45-74 \mathrm{~mm})$, and severe pain $(75-100 \mathrm{~mm})$ [15].

\section{3) The Quebec Disability Scale}

This is a condition-specific measure of disability first described by Kopec et al. [16]. The Quebec Disability Scale is a 20 -item self-administered instrument designed to assess the level of functional disability in individuals with low back pain. The patient is asked to rate the degree of his or her difficulty in performing different activities from 0 (not difficult at all) to 5 (unable to do), giving a total score ranging from 0 (no disability) to 100 (maximum disability). The reliability and validity of the original version and the Iranian version [17] have been documented.

\section{Statistical analysis}

We used PASW ver. 18.0 (SPSS Inc., Chicago, IL, USA), for all data analysis. Student $t$-tests or paired $t$-tests were used for continuous variables, and chi-square tests were used for categorical variables when comparing differences 
between completers in the two studied groups. All participating nursing assistants were official employees of the included hospitals and accessible after 6 months, resulting in no loss to follow up.

\section{Results}

In total, we included 125 eligible nursing assistants from the control $(n=62)$ and intervention $(n=63)$ hospitals. Most participants in the intervention $(57.1 \%, \mathrm{n}=36)$ and control $(64.5 \%, \mathrm{n}=40)$ groups were aged between 30 and 45 years, and most in the intervention $(82.5 \%, \mathrm{n}=52)$ and control $(75.8 \%, n=47)$ groups were male. The other demographic characteristics are shown in Table 1. No significant differences existed between the two groups in terms of the demographic data, including the age and gender distribution (by chi-square testing, all $p>0.05$ ).
We performed no statistical analysis regarding the effects of demographic data on the interventional program or the resulting WRLBP disability because we only aimed to investigate the effects of the educational program on pain severity and disability reduction among nursing assistants suffering from WRLBP. We wanted to show that the potential confounding effects of any demographic data were the same in each group.

Comparison of the intervention and control groups regarding preventive behavior for low back pain at baseline and at 6 months is shown in Table 2. At baseline, participants in the control group were already complying with back pain prevention behavior significantly more than those in the intervention group. However, the behavior score improved significantly by 6 months in the intervention group. Table 2 also shows that there was a significant difference between the two groups regarding their mean

Table 1. Comparison of demographic characteristics of participants of both groups

\begin{tabular}{|c|c|c|c|}
\hline Variable & Intervention (n=63) & Control (n=62) & $p$-value \\
\hline Age (yr) & & & $0.36^{\mathrm{a})}$ \\
\hline$<30$ & 14 (22.2) & $15(24.2)$ & \\
\hline $30-45$ & $36(57.1)$ & $40(64.5)$ & \\
\hline$>45$ & $13(20.6)$ & $7(11.3)$ & \\
\hline $\mathrm{BMI}$ & & & $0.97^{\mathrm{a})}$ \\
\hline$<20$ & $4(6.3)$ & $5(8.1)$ & \\
\hline $20-25$ & $22(34.9)$ & $22(35.5)$ & \\
\hline $26-30$ & $24(38.1)$ & $23(37.1)$ & \\
\hline$>30$ & $7(11.1)$ & $8(12.9)$ & \\
\hline Educational level & & & $0.26^{\mathrm{a})}$ \\
\hline Primary school & $20(31.7)$ & $11(17.7)$ & \\
\hline Secondary school & $16(25.4)$ & $22(35.5)$ & \\
\hline High school & $23(36.5)$ & $25(40.3)$ & \\
\hline Associate degree & $4(6.3)$ & $4(6.3)$ & \\
\hline Sex & & & $0.35^{\mathrm{a})}$ \\
\hline Male & $52(82.5)$ & $47(75.8)$ & \\
\hline Female & $11(17.5)$ & $15(24.2)$ & \\
\hline Sciatica pain & & & $0.65^{\text {a) }}$ \\
\hline Yes & $18(28.6)$ & $20(32.3)$ & \\
\hline No & $45(71.4)$ & $42(67.7)$ & \\
\hline Pain intensity (VAS) & $1.97 \pm 5.01$ & $2.27 \pm 5.40$ & $0.31^{\mathrm{b})}$ \\
\hline Disability (Qubece score) & $27.63 \pm 20.74$ & $32.27 \pm 27.43$ & $0.21^{\mathrm{bl}}$ \\
\hline
\end{tabular}

Values are presented as number (\%) or mean \pm standard deviation. VAS, visual analogue scale.

${ }^{\text {a) }}$ Chi-square; ${ }^{\text {bl }}$ t-test. 
Table 2. Comparison of the two groups regarding low back pain preventive behavior at baseline and 6-month follow-up

\begin{tabular}{|c|c|c|c|}
\hline \multirow{2}{*}{ Variable } & \multicolumn{2}{|c|}{ Behavior score } & \multirow{2}{*}{$p$-value ${ }^{\mathrm{a}}$} \\
\hline & Intervention group ( $n=63$ ) & Control group ( $\mathrm{n}=62$ ) & \\
\hline Baseline & $30.81 \pm 8.35$ & $34.37 \pm 7.16$ & $<0.01$ \\
\hline 6-Month follow-up & $34.22 \pm 6.55$ & $29.87 \pm 6.507$ & $<0.001$ \\
\hline$p$-value ${ }^{\mathrm{b})}$ & $<0.001$ & $<0.001$ & \\
\hline
\end{tabular}

Values are presented as mean \pm standard deviation.

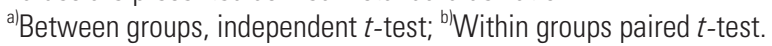

Table 3. Comparison of the two groups regarding pain intensity and disability before and after the intervention

\begin{tabular}{|c|c|c|c|}
\hline Variable & Intervention group ( $\mathrm{n}=63$ ) & Control group ( $\mathrm{n}=62$ ) & $p$-value ${ }^{\mathrm{a}}$ \\
\hline \multicolumn{4}{|c|}{ Pain intensity (VAS score) } \\
\hline Baseline & $5.01 \pm 1.97$ & $5.40 \pm 2.27$ & 0.31 \\
\hline 6-Month follow-up & $3.42 \pm 2.53$ & $5.03 \pm 2.13$ & $<0.001$ \\
\hline$p$-value ${ }^{\mathrm{b})}$ & $<0.001$ & 0.22 & \\
\hline \multicolumn{4}{|l|}{ Disability } \\
\hline Baseline & $27.63 \pm 20.74$ & $32.27 \pm 27.43$ & 0.21 \\
\hline 6-Month follow-up & $21.33 \pm 19.37$ & $30.33 \pm 18.03$ & 0.07 \\
\hline$p$-value $\mathrm{e}^{\mathrm{b})}$ & 0.23 & 0.43 & \\
\hline
\end{tabular}

Values are presented as mean \pm standard deviation.

VAS, visual analogue scale.

a)Between groups, independent $t$-test; ${ }^{b}$ Within groups paired $t$-test.

scores for low back pain preventive behaviors at 6 months (Table 2).

Table 3 summarizes the comparison of the two groups regarding pain intensity and disability at baseline and 6 months. As shown, there were no differences in pain intensity between the groups at baseline $(p=0.31)$, but that there was a significant difference by 6 months $(p<0.001)$. Specifically, participants in the control group experienced more severe pain after 6 months. Within-group analysis showed that there was a significant difference between baseline and 6 months in terms of pain intensity in the intervention group $(p<0.001)$, but that there was no significant improvement in pain severity in the control group $(p=0.22)$.

\section{Discussion}

After a 6-month period, we showed that a multidisciplinary workplace educational intervention with ergonomic posture training that is based on social cognitive theory can decrease the intensity of back pain among nursing assistants by increasing preventive behaviors. This indicates that such an approach, focusing on ergonomic posture training and promoting self-efficacy and selfregulation, can help nursing assistants maintain healthy behaviors for vertebral biomechanics when caring for or transferring patients.

Moreover, we believe that focusing on the values and outcomes of maintaining ergonomic vertebral postures and enhancing the emotional coping strategies used by nursing assistants after implementing preventive behaviors at work probably contributes to continued adoption of healthy behaviors up to six months. Consistent with the work by Kirk et al. [18], use of these behavior change processes appeared to result in the workers advancing to the next stage of preventive behavior to reduce WRLBP. Similarly, other research has shown that complying with correct vertebral biomechanics during work can reduce the intensity of low back pain [19]. We showed that a multidisciplinary educational and training intervention can significantly improve low back pain severity in nursing assistants up to 6 months after baseline assessment. This 
supports the study by Rasmussen [20], who demonstrated that a multifaceted intervention consisting of participatory ergonomics, physical training, and cognitive-behavioral training can reduce the number of days with low back pain as well as the intensity of WRLBP among workers, primarily nurse aides, in nursing and care homes [20]. The intensity of low back pain is a significant risk factor for sickness absence [21], so the reduction in pain intensity in our study can be beneficial for both employees and employers. Consistent with our findings, previous research conducted in a rehabilitation center verified that three theory-based treatments including cognitive-behavioral, physical, or both) were more effective than remaining on a waiting list for reducing patients' functional limitations, main complaints, and pain intensity [22]. However, in contrast with previous research, we did not show a significant improvement in disability, which may be due to the short duration and low intensity of our intervention. However, Gohner and Schlicht [23] did report that a short and inexpensive cognitive-behavioral training program was an effective intervention that could enable patients with back pain to follow treatment recommendations on a regular basis, even though no group differences emerged regarding pain intensity in their study. Studies of other models in Iran have also achieved positive results. For instance, Zeidi et al. [19] showed that developing an ergonomics educational program based on a transtheoretical model considerably enhanced adaptation to correct body posture among computer users. As with previous studies [22], we suggest that further research is needed to investigate the effects of theory-based treatments and confirm our findings if we are to develop more effective treatments.

Several studies have investigated the effectiveness of different treatment protocols to decrease musculoskeletal disorders (especially WRLBP) in nurses. These include introducing education programs, ergonomic changes in work settings, relaxation programs, exercise programs, and multidisciplinary team approaches [22]. However, there have been very few interventional trials to assess the effectiveness of the multidisciplinary approaches for preventing and reducing WRLBP and its consequences in workplaces with physically demanding work. When done, such studies have tended to be unsuccessful [20]. Poor body mechanics and lack of training in appropriate lifting techniques have been considered the main cause of the high prevalence of upper and lower back pain by preoperative nurses and technicians [10].
There is a high prevalence of low back pain among nurse aides, and it is known that multidisciplinary ergonomics or theory-based educational interventions are important for reducing back pain. Despite this, and to the best of our knowledge, we are not aware of any educational interventions being developed for Iranian nursing assistants based on social cognitive theory. Considering the breadth of the field of low back pain, maybe current educational programs on this issue are insufficient for this specific population. Therefore, further theory-based education is needed to improve the prevention of low back pain among nursing assistants through in-service training. We recommend that nurse administrators use the evidence in this study to advocate for more educational programs to be funded and for those programs to be sustained over time.

In addition to the strengths of this study, there is some limitation that may affect the results. One such limitation is that we performed no paraclinical tests when diagnosing chronic mechanical low back pain or assessing whether patients met the exclusion criteria. Using self-report questioner is other limitation that might make bias for collected data. Furthermore, compliance with the intervention was self-reported and may have been overstated. However, we did use a control group with the same age distribution to account for the possible effects of any confounders. In Iran, many people with WRLBP are not treated and nursing aids are usually a neglected group. Therefore, it is important that we have shown the efficacy of a well-designed educational intervention over non-treatment. This approach can easily be inserted into the healthcare system of Iran to reduce pain, disability, and absenteeism among nurse aides. We recommend comparing our program with other interventions in future studies.

\section{Conclusions}

In this study, we showed that a workplace-based educational intervention can reduce the intensity of low back pain among nurse aides. Thus, our data indicate that these interventions are suitable for improving WRLBP among nursing assistants working in hospital settings.

\section{Conflict of Interest}

No potential conflict of interest relevant to this article was reported. 


\section{References}

1. Karahan A, Kav S, Abbasoglu A, Dogan N. Low back pain: prevalence and associated risk factors among hospital staff. J Adv Nurs 2009;65:516-24.

2. Poitras S, Blais R, Swaine B, Rossignol M. Management of work-related low back pain: a populationbased survey of physical therapists. Phys Ther 2005; 85:1168-81.

3. Yip VY. New low back pain in nurses: work activities, work stress and sedentary lifestyle. J Adv Nurs 2004; 46:430-40.

4. Bos E, Krol B, van der Star L, Groothoff J. Risk factors and musculoskeletal complaints in non-specialized nurses, IC nurses, operation room nurses, and X-ray technologists. Int Arch Occup Environ Health 2007; 80:198-206.

5. Verbeek JH, Martimo KP, Kuijer PP, Karppinen J, Viikari-Juntura E, Takala EP. Proper manual handling techniques to prevent low back pain, a Cochrane systematic review. Work 2012;41 Suppl 1:2299-301.

6. Tullar JM, Brewer S, Amick BC 3rd, et al. Occupational safety and health interventions to reduce musculoskeletal symptoms in the health care sector. J Occup Rehabil 2010;20:199-219.

7. Holtermann A, Clausen T, Jorgensen MB, Burdorf A, Andersen LL. Patient handling and risk for developing persistent low-back pain among female healthcare workers. Scand J Work Environ Health 2013;39: 164-9.

8. Owen BD, Keene K, Olson S. An ergonomic approach to reducing back/shoulder stress in hospital nursing personnel: a five year follow up. Int J Nurs Stud 2002;39:295-302.

9. Hsiao YC, Chen MY, Gau YM, Hung LL, Chang SH, Tsai HM. Short-term effects of a health promotion course for Taiwanese nursing students. Public Health Nurs 2005;22:74-81.

10. Sheikhzadeh A, Gore C, Zuckerman JD, Nordin M. Perioperating nurses and technicians' perceptions of ergonomic risk factors in the surgical environment. Appl Ergon 2009;40:833-9.

11. Sarallah S, Sadat TS, Jamshidi AR, Joan W. A multidisciplinary work-related low back pain predictor questionnaire: psychometric evaluation of Iranian patient-care workers. Asian Spine J 2016;10:501-8.

12. Glanz K, Rimer BK, Viswanath K. Health behavior and health education: theory, research, and practice. San Francisco: John Wiley \& Sons; 2008.

13. Rogers B, Ostendorf J. Occupational health nursing. New York: John Wiley \& Sons; 2001.

14. Joyce CR, Zutshi DW, Hrubes V, Mason RM. Comparison of fixed interval and visual analogue scales for rating chronic pain. Eur J Clin Pharmacol 1975;8: 415-20.

15. Jensen MP, Chen C, Brugger AM. Interpretation of visual analog scale ratings and change scores: a reanalysis of two clinical trials of postoperative pain. J Pain 2003;4:407-14.

16. Kopec JA, Esdaile JM, Abrahamowicz M, et al. The Quebec Back Pain Disability Scale. Measurement properties. Spine (Phila Pa 1976) 1995;20:341-52.

17. Mousavi SJ, Akbari ME, Mehdian H, et al. Low back pain in Iran: a growing need to adapt and implement evidence-based practice in developing countries. Spine (Phila Pa 1976) 2011;36:E638-46.

18. Kirk A, MacMillan F, Webster N. Application of the transtheoretical model to physical activity in older adults with type 2 diabetes and/or cardiovascular disease. Psychol Sport Exerc 2010;11:320-4.

19. Zeidi IM, Morshedi H, Zeidi BM. The effect of interventions based on transtheoretical modelling on computer operators' postural habits. Clin Chiropractic 2011;14:17-28.

20. Rasmussen CD, Holtermann A, Bay H, Sogaard K, Birk Jorgensen M. A multifaceted workplace intervention for low back pain in nurses' aides: a pragmatic stepped wedge cluster randomised controlled trial. Pain 2015;156:1786-94.

21. van den Heuvel SG, Ariens GA, Boshuizen HC, Hoogendoorn WE, Bongers PM. Prognostic factors related to recurrent low-back pain and sickness absence. Scand J Work Environ Health 2004;30:459-67.

22. Smeets RJ, Vlaeyen JW, Hidding A, et al. Active rehabilitation for chronic low back pain: cognitivebehavioral, physical, or both? First direct posttreatment results from a randomized controlled trial [ISRCTN22714229]. BMC Musculoskelet Disord 2006;7:5.

23. Gohner W, Schlicht W. Preventing chronic back pain: evaluation of a theory-based cognitive-behavioural training programme for patients with subacute back pain. Patient Educ Couns 2006;64:87-95. 